\title{
Unilateral Internal Cerebral Vein Thrombosis: Case Reports and Review of the Literature
}

\author{
Ashley Hu ${ }^{1}$ (D) ${\text { Mariam } \text { Thomas }^{2} \text { (D) } \cdot \text { Saadia Chaudhary }}^{3}$ - Gasser Hathout ${ }^{1,2,4}$
}

Accepted: 4 January 2021 / Published online: 18 January 2021

(C) The Author(s) 2021

\begin{abstract}
The purpose of this manuscript is to present two cases of unilateral internal cerebral vein thrombosis (UICVT) and use them to review the typical imaging findings and clinical presentation, provide a differential diagnosis when presented with a unilateral thalamic lesion, and discuss current literature findings of internal cerebral vein thrombosis, including the interesting trend of leftsided involvement. Two cases of UICVT were gathered from our institutions. A literature review was then conducted, and cases in the literature were compared to look for common features. Both of our cases involved the left internal cerebral vein. A total of 7 cases of unilateral and 8 cases of bilateral internal cerebral vein thrombosis were reviewed from the literature. Of the unilateral cases, all but one were on the left, and the one on the right was in a patient with right-sided malignancy after surgical resection. Most of these cases completely resolved with anticoagulation. UICVT is an extremely rare entity, with less than 10 cases reported in the literature. The prognosis of this disease is excellent if diagnosed and treated early; however, with delayed diagnosis and treatment, this process can be fatal. This highlights the importance of keeping this diagnosis on the differential when presented with a unilateral thalamic lesion.
\end{abstract}

Keywords Radiology $\cdot$ Stroke $\cdot$ Cerebral venous thrombosis

\section{Introduction}

Cerebral vein thrombosis is an uncommon cause of stroke, with an estimated incidence ranging from 0.2 to 1.3 cases per 100,000 per year [1]. Of these, only an estimated $11 \%$ involves the deep cerebral veins [2]. The deep cerebral veins are comprised of the internal cerebral veins, basal veins of Rosenthal, and vein of Galen. When internal cerebral vein thrombosis occurs, the most common finding is bilateral thalamic infarction, with possible involvement of the adjacent

This article is part of the Topical Collection on Imaging

Ashley Hu

ashleyhu@mednet.ucla.edu

1 Department of Radiology, UCLA Medical Center, Los Angeles, CA, USA

2 Department of Radiology, Olive View-UCLA Medical Center, Sylmar, CA, USA

3 Department of Radiology, Huntington Memorial Hospital, Pasadena, CA, USA

4 Department of Radiology, Greater Los Angeles VA Medical Center, Los Angeles, CA, USA basal ganglia. There have also been cases of unilateral thalamic infarction, but still in the setting of bilateral internal cerebral vein thrombosis. Few cases of unilateral internal cerebral vein thrombosis are seen, with only a handful published in the literature. Here, we will present two cases of unilateral internal cerebral vein thrombosis (UICVT), and use them to review the typical imaging findings and clinical presentation, provide a differential diagnosis when presented with a unilateral thalamic lesion, and discuss current literature findings of internal cerebral vein thrombosis, including the interesting trend of left-sided involvement.

\section{Methods}

Two cases of unilateral internal cerebral vein thrombosis were gathered from our institutions. For the literature review, we searched our institution's library catalog, which includes multiple databases including MEDLINE, for "internal cerebral vein thrombosis" along with the terms "unilateral" and "bilateral," separately. Multiple cases were collected and logged to include each patient's age, gender, risk factors, presenting symptoms, treatment received, vessels thrombosed as seen on imaging, and ultimate outcome. 


\section{Results}

\section{Case 1}

A 63-year-old female presented with dysarthria and right hemiparesis. She had no other complaints and denied symptoms of altered mental status, headache, nausea, or vision changes. She had a history of hypertension, vertigo, and colorectal cancer with oligometastasis to the liver, receiving active chemotherapy treatments at the time of presentation. Physical examination was significant for mild dysarthria and right upper extremity pronator drift.

The initial CT findings demonstrated hypodensity of the left thalamus and posterior limb of the internal capsule (Fig. 1a). MRI confirmed the presence of edema in the left thalamus, with a small amount of diffusion restriction in the ventromedial thalamus (Fig. 1c). No enhancement of the lesion was seen (Fig. 1e). The initial impression of the CT was infarct versus tumor. When the MR was obtained, it was noted that there was non-enhancement of the left internal cerebral vein (not shown), raising concern for a rare unilateral internal cerebral vein thrombosis. A CT angiogram (CTA) was obtained for confirmation and demonstrated thrombosis of the left
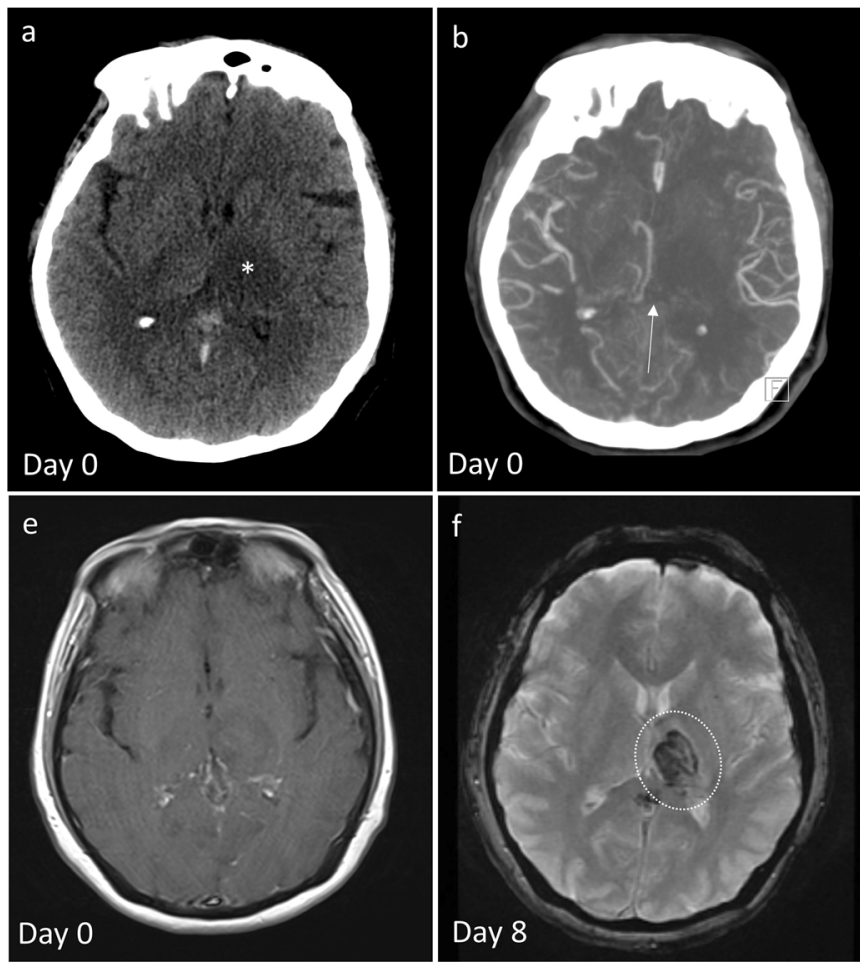

Fig. 1 a Noncontrast $\mathrm{CT}$ of the head demonstrates hypoattenuation in the region of the left thalamus (asterisk). Day 0 represents the day of the initial presentation. b CT angiogram obtained after a $45 \mathrm{~s}$ delay following contrast administration demonstrates no arterial filling defect, but an incidental absence of filling in the left internal cerebral vein (arrow). c MRI FLAIR sequence demonstrates focal hyperintensity of the thalamus (circle). d Initial gradient echo images demonstrate susceptibility artifact in the left internal cerebral vein and vein of Galen internal cerebral vein, thalamostriate vein, vein of Galen, and straight sinus (Fig. 1b). The patient was started on a heparin drip, with a transition to enoxaparin $60 \mathrm{mg}$ BID prior to discharge. Nine days after the initial presentation, MRI showed T2/FLAIR signal hyperintensity and blooming artifact in the left thalamus compatible with hemorrhage (Fig. 1f). The left internal cerebral vein, vein of Galen, and straight sinus continued to show an absence of flow. On day 12, CT of the head showed a decrease in the amount of left thalamic hemorrhage. Clinically, the patient's symptoms had resolved, and on day 13, she was discharged from the hospital.

\section{Case 2}

A 49-year-old female with no significant past medical history presented with altered mental status, right-sided hemiparesis, and aphasia. Pertinent negatives included no headache, nausea, or vision changes. The patient reportedly had a severe upper respiratory infection approximately 5 days prior to presentation. Pertinent neurological examination was significant for $1 / 5$ strength in the right upper extremity, equal strength in the bilateral lower extremities, and upgoing right Babinski reflex. The patient was awake but non-verbal and unable to
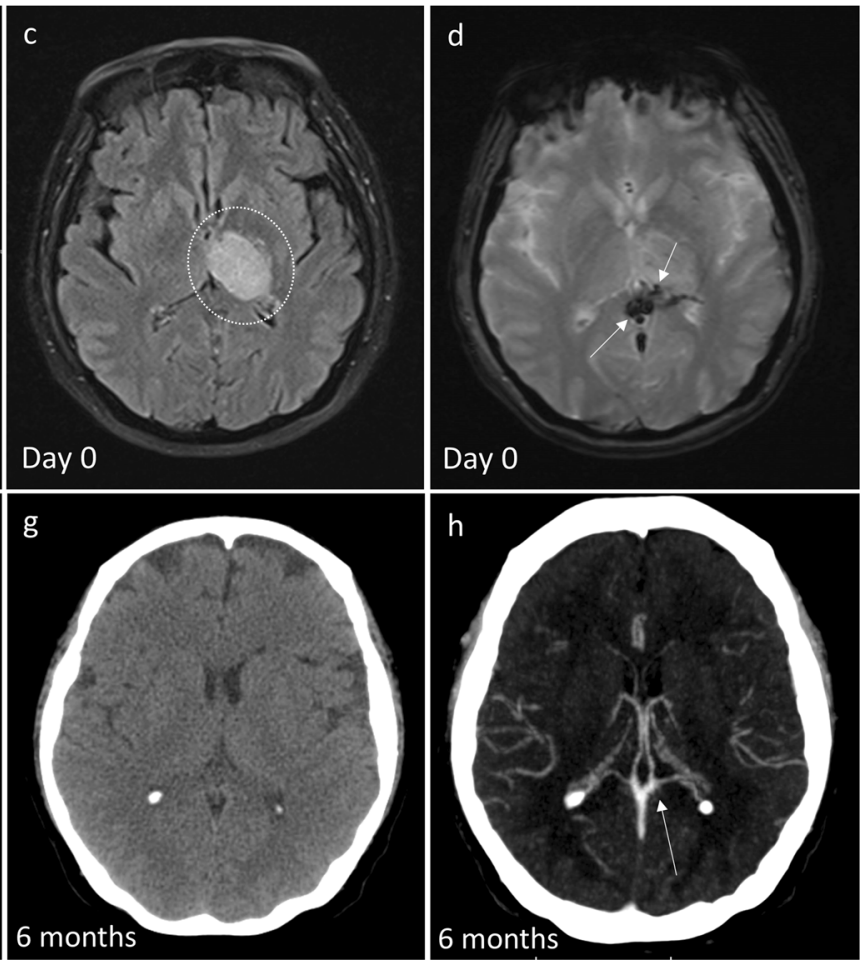

(arrows), compatible with the thrombus. e MRI post-contrast images demonstrate no significant enhancement of the thalamic lesion. $\mathbf{f}$ Gradient echo images from an MRI done at a later date demonstrated developing hemorrhage in the region of infarct (circle). $g$ Noncontrast $\mathrm{CT}$ done at 6 months after the initial presentation demonstrates resolution of thalamic hypoattenuation. $\mathbf{h}$ CT angiogram done at 6 months demonstrates patency of the previously thrombosed left internal cerebral vein (arrow) 
follow commands. Labs were significant for a hemoglobin level of $6.7 \mathrm{mg} / \mathrm{dL}$, which was attributed to menorrhagia noted on physical exam.

The initial CT scan demonstrated decreased attenuation in the left thalamus and left basal ganglia (Fig. 2a). On MRI, an extensive infiltrative $\mathrm{T} 1$ hypoattenuating and $\mathrm{T} 2$ hyperattenuating lesion was seen involving the left thalamus (Fig. 2b). In this lesion were also areas of diffusion restriction and susceptibility representing microhemorrhage (Fig. 2d). Just as with the prior case, no enhancement was seen (Fig. 2c). The leading initial differential diagnosis after both the $\mathrm{CT}$ and the MRI was infiltrative tumor. MR angiogram on hospital day two demonstrated a paucity of flow in the bilateral internal cerebral veins, left greater than right, vein of Galen, and straight sinus. CT venogram (CTV) done on the same day for further clarification showed occlusion of the left internal cerebral vein, vein of Galen, straight sinus, and right sigmoid sinus and jugular bulb (Fig. 2e). The patient was started on enoxaparin $60 \mathrm{mg}$ BID the same day. On hospital day 10, MRI showed that the left internal cerebral vein remained occluded with a larger amount of diffusion restriction within the left thalamus, concerning for increased size of infarction (not shown). Vasogenic edema was noted to be decreased. On hospital day 13, the patient was discharged to an acute rehabilitation facility with limited improvement. Approximately 3 weeks later, she was discharged from acute rehabilitation with her clinical symptoms resolved.

\section{Literature Review}

A summary of the cases obtained through the literature is provided in Tables 1 and 2 .

A total of 7 cases of UICVT were found in the literature. Of these, 6 out of 7 were on the left side. Five out of 7 used anticoagulation to treat the condition, all with reported positive outcomes, described as either resolution of symptoms, resolved thalamic edema, or some degree of recanalization. No common risk factors were identified.
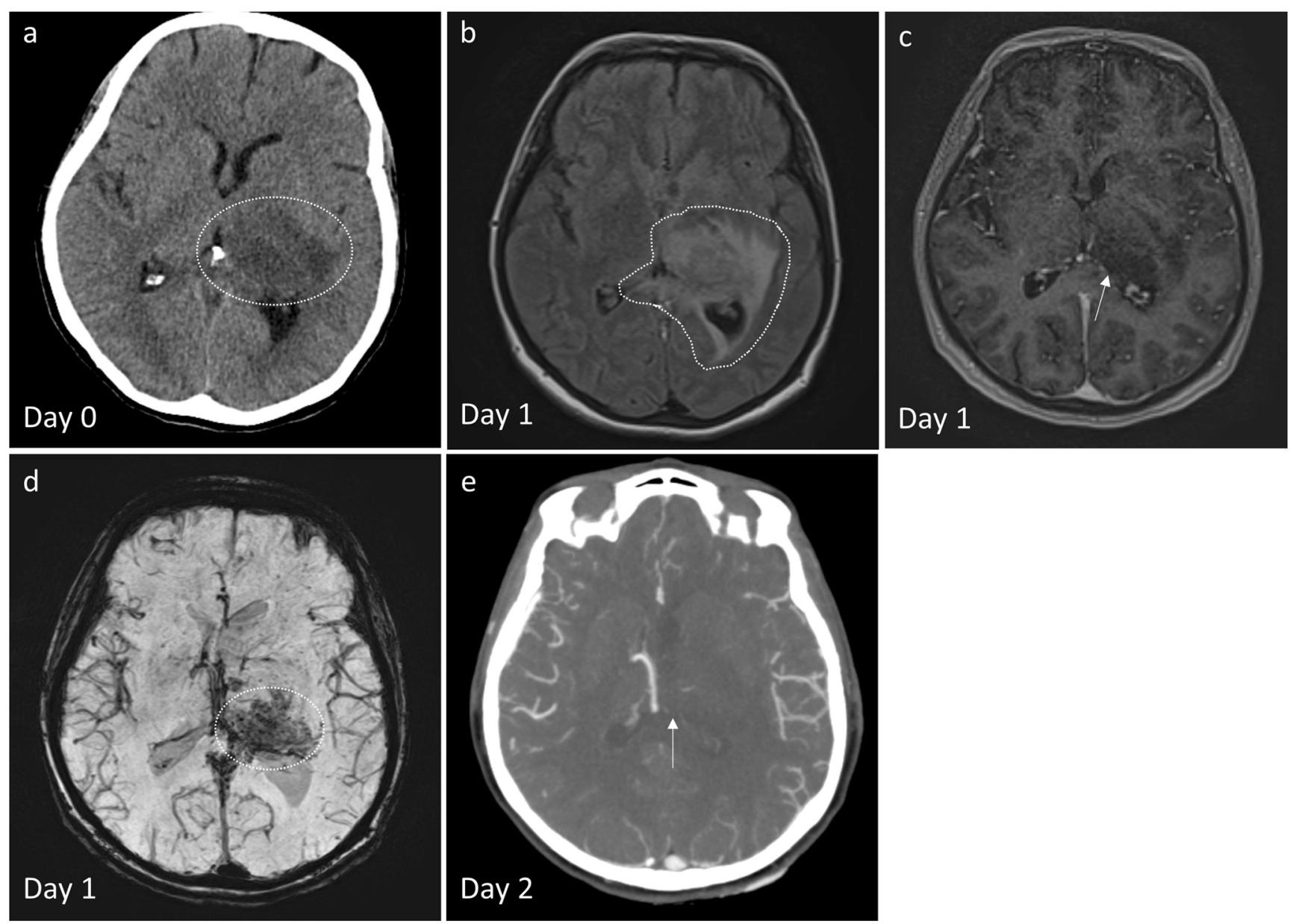

Fig. 2 a Noncontrast axial CT of the head demonstrates hypoattenuation in the region of the left thalamus and basal ganglia (circle). b MRI FLAIR sequence demonstrates edema in the left thalamus extending to the adjacent white matter, corpus callosum, and periventricular region (outlined area). c Post-contrast sequence demonstrates a lack of

enhancement in the left thalamus (arrow). d Susceptibility-weighted imaging demonstrates a microhemorrhage in the left thalamus (circle). e CT venogram demonstrates a filling defect in the left internal cerebral vein (arrow) 


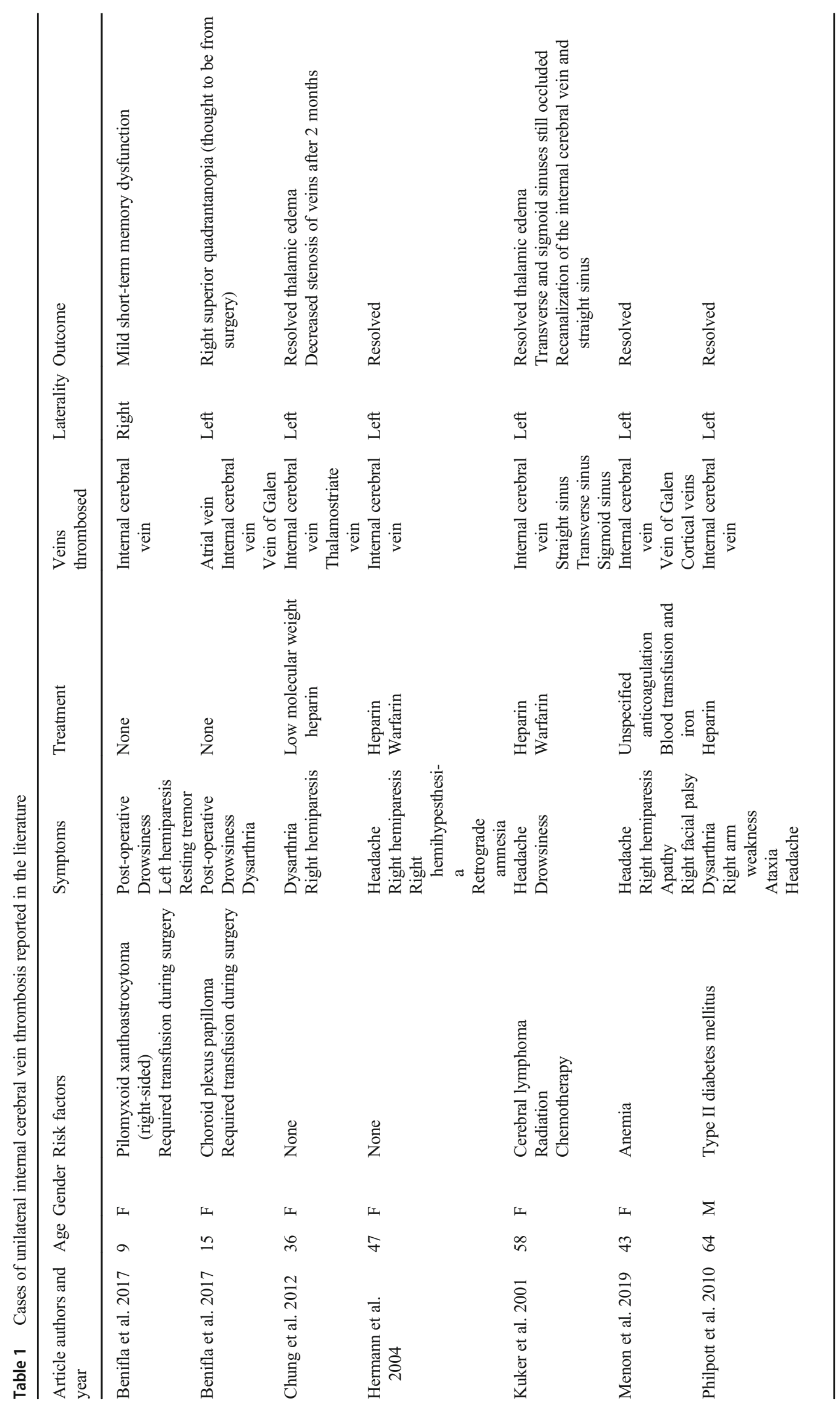




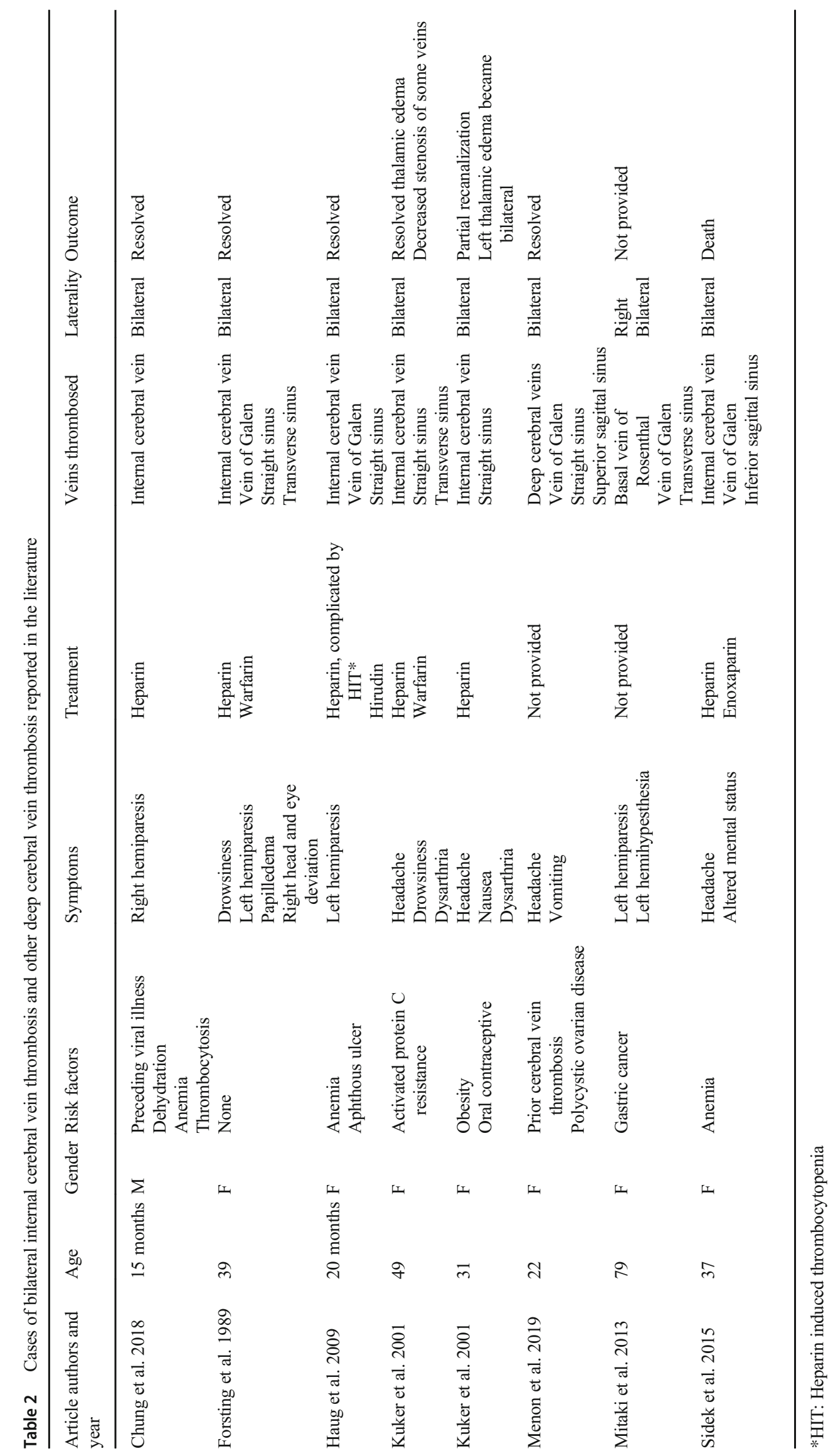


Table 3 Advantages and disadvantages of different imaging modalities in diagnosing deep cerebral vein thrombosis

\begin{tabular}{|c|c|c|}
\hline Imaging modality & Advantages & Disadvantages \\
\hline Non-contrast CT & $\begin{array}{l}\text { Almost always obtained when patient presents } \\
\text { with focal neurological deficits } \\
\text { May show thalamic hypoattenuation/edema }\end{array}$ & $\begin{array}{l}\text { Inability to visualize vessels } \\
\text { Sensitivity of detecting a hyperdense thrombosed } \\
\text { vessel is only } 50 \% \\
\text { Radiation }\end{array}$ \\
\hline $\mathrm{CT}$ angiogram & Usually ordered as part of the stroke workup & $\begin{array}{l}\text { Timing not tailored for venous structures } \\
\text { Radiation }\end{array}$ \\
\hline CT venogram & High sensitivity (95-96\%) & $\begin{array}{l}\text { Requires high suspicion for venous thrombosis, } \\
\text { and is not routinely done } \\
\text { Radiation }\end{array}$ \\
\hline MRI & $\begin{array}{l}\text { Multiple sequences to characterize the nature of } \\
\text { thalamic "lesion" } \\
\text { Thrombus may be seen on gradient echo or } \\
\text { susceptibility-weighted sequences } \\
\text { No ionizing radiation }\end{array}$ & No dedicated vessel imaging \\
\hline MR venogram & $\begin{array}{l}\text { High sensitivity }(95-96 \%) \\
\text { No ionizing radiation }\end{array}$ & Spatial resolution is inferior to CT venogram \\
\hline
\end{tabular}

A total of 8 cases of bilateral internal cerebral vein thrombosis were identified in the literature. In these cases, 6 out of 8 outlined how they were treated with anticoagulation. Four cases reported full resolution of symptoms, while 1 case reported an outcome of death. Three of the cases had anemia demonstrated in labs.

\section{Discussion}

Thrombosis of the cerebral venous system is an uncommon cause of stroke, accounting for approximately 0.2 to 1.3 stroke cases per 100,000 per year [1]. Of these, only an estimated $11 \%$ involves the deep cerebral veins [2]. Thrombosis of the internal cerebral veins is rare, but when it does occur, it is most often bilateral. UICVT is an extremely rare entity with less than 10 reported cases in the literature (Table 1). Interestingly, of the reported cases in the literature, all but one case were left sided. The one case that presented on the right was in the postoperative setting after resection of a right-sided tumor. [3]. In the two cases that we presented, both were also on the left. Some articles have also found that when there is bilateral internal cerebral vein thrombosis, thalamic infarct preferentially occurs on the left [4-6]. While the exact pathophysiology of unilateral thrombosis is not yet fully understood, a theory has been proposed by Philpott et al. suggesting that the underlying asymmetry in venous drainage may play a role, with the right-sided deep venous system draining better than the left [7]. This may mirror the well-known asymmetry where the superior sagittal sinus drains preferentially into the right transverse sinus [8]. Additionally, there has been speculation as to whether hemispheric dominance may play a role, causing right-sided internal cerebral vein thrombosis to be clinically silent, in contradistinction to left-sided internal cerebral vein thrombosis. Our working hypothesis is that the cause of unilateral thrombosis and left-sided predilection is likely due to asymmetric drainage as suggested by Philpott et al., but the anatomic verification of this has yet to be studied and elucidated.

Among the reported cases, the likely etiologies of deep cerebral vein thrombosis appear similar to those of superficial venous thrombosis. Our cases demonstrated thrombosis in the setting in malignancy and anemia. In addition to these, others have described deep cerebral vein thrombosis in the setting of diabetes mellitus, oral contraceptive use, hypercoagulable state, and a post-operative state [3-7, 9-12] (Tables 1 and 2). Still, others have had no known past medical history [13-15]. However, it is difficult to say whether these conditions are the etiology or if they are simply associated with thrombosis. Despite this, physicians and radiologists can use this understanding to have a higher level of suspicion for cerebral vein thrombosis when presented with certain patient histories.

The presentation of deep cerebral vein thrombosis can present as focal or generalized neurologic symptoms. Some of the symptoms can be derived from the function of the territories that are drained by the deep cerebral veins. These include the thalamus, basal ganglia, deep structures of the inferior frontal, parietal and temporal lobes, and corpus callosum [16]. Notably, however, as seen in our cases, venous occlusion most often affects the thalamus, causing findings of thalamic infarction such as edema and microhemorrhage. The thalamus is known to act as a relay center for sensory and motor processing [17]. As a result, it is not surprising that the symptoms of internal cerebral vein thrombosis, as seen in our cases and in the literature, include hemiparesis and hemihypesthesia. 
The thalamus is also known to be involved in language processing and level of alertness [18, 19], which correlates nicely with the dysarthria, word finding difficulty, and drowsiness seen in our review. This constellation of symptoms, if identified in the clinical setting, could direct the treatment physician and radiologist towards this diagnosis of internal cerebral vein thrombosis, especially if arterial stroke workup is negative.

When a patient is worked up for neurological deficits, labs are universally obtained to seek out any significant abnormality that might contribute to the patient's symptoms. Typically, labs are unrevealing. However, with the right clinical suspicion, more specific labs can be drawn and sent for workup of any underlying hypercoagulability syndrome. One interesting lab abnormality that was seen in multiple cases of internal cerebral vein thrombosis was anemia $[4,6,12,14]$. In our analysis, we found that they all had a hemoglobin level of less than $7.0 \mathrm{~g} / \mathrm{dL}$. Some studies in the literature stating that there is some association between anemia and a thrombotic diathesis [20]. Others have also found an increased association of anemia with cerebral venous thrombosis specifically $[1,21]$.

In order to make the correct diagnosis, different imaging modalities can be used, all with their own advantages (Table 3). Given that most of these patients present with stroke-like symptoms, the initial scan will presumably be a non-contrast CT of the head. Depending on the degree of edema, the affected thalamus can be hypodense and swollen compared to the other side. Occasionally, a hyperdensity in the region of the thrombosed vein may be seen, akin to the "hyperdense MCA sign." However, the sensitivity of this finding is only about 50\% [22]. As the most common clinical suspicion is arterial stroke, CTA generally follows. While the timing is not as optimized as CTV, occasionally, CTA can demonstrate the absence of filling of the internal cerebral veins and other deep venous structures, as in our cases. CTV will also show these filling defects, with contrast timing more tailored to the veins. The sensitivity of detecting venous thrombosis on CTV approaches $95-96 \%$ according to some reports $[22,23]$ and thus is a very reliable study for detecting cerebral vein thrombosis. One study that compared CTV side by side with digital subtraction angiography showed that CTV had a sensitivity ranging from 55 to $100 \%$ depending on the vein in question and had an overall sensitivity of 95\% [24]. MRI of the brain will demonstrate T2 and FLAIR hyperintensity in the involved thalamus, again suggestive of thalamic edema. Other structures in the ipsilateral basal ganglia may also be involved. On GRE sequences, initial scans may show susceptibility artifact in the thrombosed deep cerebral veins. In addition, over the course of a few days, thalamic microhemorrhage may develop. MR venography (MRV), with contrast or with time-of-flight technique, is comparable to CTV, also with 95-96\% sensitivity for such entities as dural sinus thrombosis [22]. In our experience, time-of-flight MRV can detect deep cerebral vein thrombosis when it involves the bilateral internal cerebral veins, vein of Galen, and or straight sinus. However, we raise the concern that the spatial resolution of time-of-flight MRV may be insufficient to detect isolated unilateral cerebral vein thrombosis. Therefore, we recommend CTV in the setting of unilateral thalamic edema.

When presented with imaging revealing a unilateral thalamic lesion, there are several differential diagnoses that come

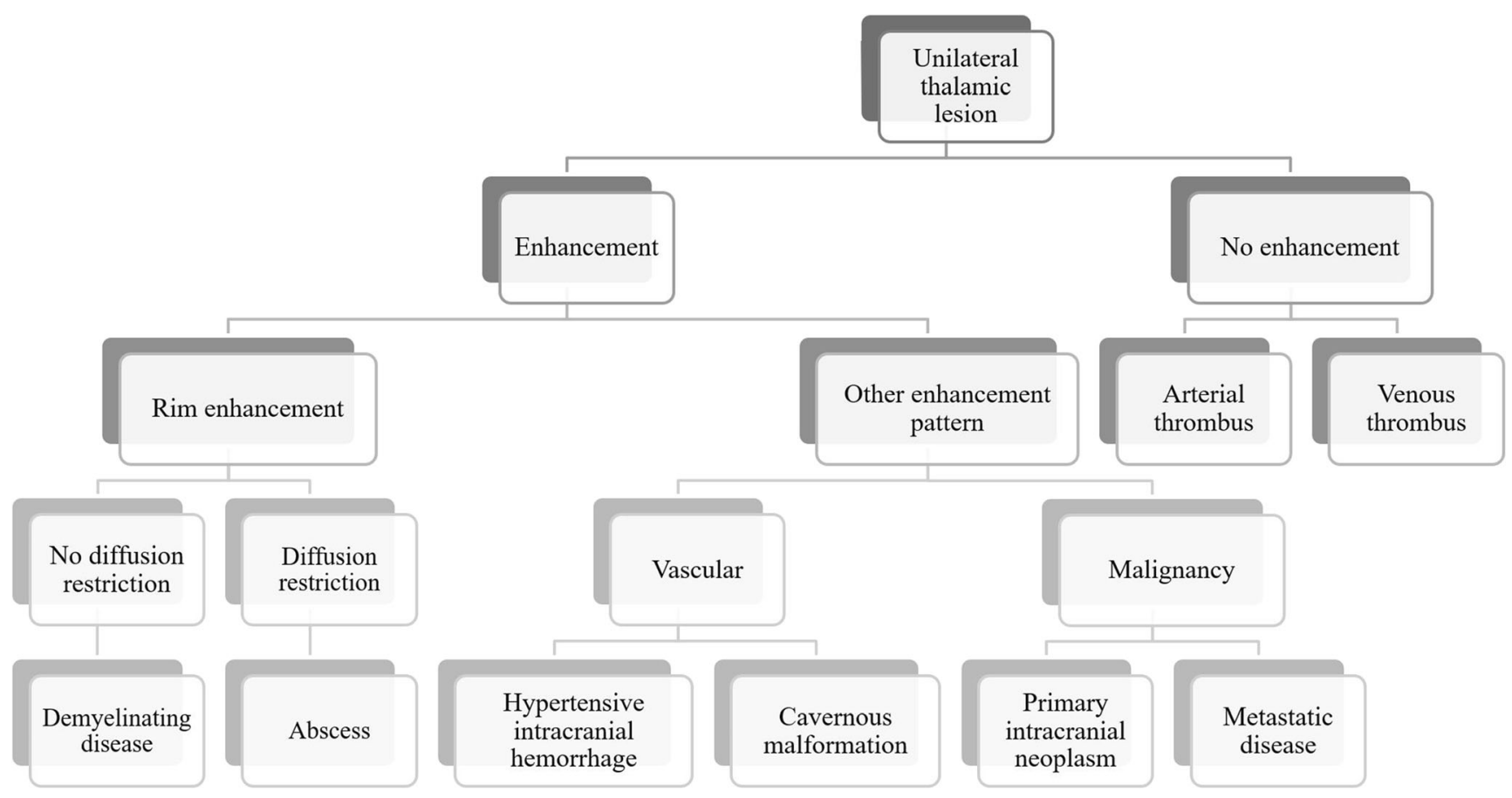

Fig. 3 Flow chart demonstrating one approach to the differential diagnosis of a unilateral thalamic lesion 
to mind (Fig. 3). The main considerations are malignancy, infection, and arterial thrombus. Less commonly, other entities such as demyelinating disease or vascular malformations may also be considered. Several key characteristics can help with the process of differentiation, which include enhancement, vessel patency, and clinical findings.

Enhancement is useful in differentiating malignancy and infection from thalamic infarct, as the former two tend to enhance. With malignancy, high-grade lesions often enhance, while low-grade lesions do not, since they are not aggressive enough to break down the blood-brain barrier. For the lower grade lesions, vessel-specific imaging such as angiography or venography is then helpful to determine whether flow to the lesion is maintained. In terms of infection, clinical history and lab values are key. In the case of abscess formation, ring enhancement and diffusion restriction are more suggestive of infection rather than venous thrombosis. Organisms that tend to favor the thalamus include certain viruses such as influenza A, parainfluenza, and atypical bacteria such as mycoplasma and tuberculosis [25].

Vessel-specific imaging is also critical for the evaluation of arterial versus venous thrombus. Differentiating arterial from venous thrombus in the thalamus requires some familiarity with arterial supply and venous drainage of the thalamus. The thalamus is primarily supplied by the thalamoperforating arteries, thalamogeniculate arteries, and posterior choroidal arteries, all of which arise from the posterior cerebral artery [25]. As a result, following the posterior cerebral artery on the CTA can help determine the patency of those vessels. On the other hand, the internal cerebral veins are formed by the confluence of the choroidal and thalamostriate veins. The internal cerebral veins converge with the basal veins of Rosenthal to form the vein of Galen, which then converges with the inferior sagittal sinus to form the straight sinus. By understanding the course of the arteries and veins of the thalamus, it becomes easier to understand and find where exactly the filling defect is located, when one is seen.

Treatment guidelines specifically tailored towards unilateral internal cerebral vein thrombosis have not been established, likely due to the paucity of cases. However, treatment generally follows the same guidelines as for any type of cerebral venous thrombosis, with unfractionated heparin or low molecular weight heparin for a duration of 3 to 12 months $[2,26]$. UICVT cases published in the literature have generally followed these recommendations. The few exceptions were those that were related to post-operative thrombosis in children, in which recovery was made without any pharmacological intervention [3]. The choice of anticoagulation varied between papers, but included the recommended therapies of either heparin or low molecular weight heparin, such as enoxaparin. After treatment, most cases described the resolution of symptoms or thalamic edema on imaging. As a result, prognosis generally appears to be favorable with appropriate and timely treatment. The two post-operative cases in which no anticoagulation was given had outcomes with mild residual deficits, which the paper stated could be attributed to the surgeries.

Our current knowledge of unilateral internal cerebral vein thrombosis is limited, but it is slowly growing as more cases are gathered and reported in the literature. While indeed rare, it is a diagnosis that the radiologist must keep in mind when approaching a unilateral thalamic lesion, because it is often initially misdiagnosed. Timely diagnosis ensures that treatment will not be delayed. As seen with the cases we reviewed, when appropriate treatment is initiated, the prognosis is generally favorable. In the future, when enough cases are gathered, perhaps further studies can be conducted in a retrospective manner to understand more about the etiology, risk factors, and left-sided involvement.

Authors' Contributions Dr. Chaudhary and Dr. Hathout provided the two case reports. All four authors contributed equally to the literature review, manuscript drafting, editing, and final approval.

Data Availability Not applicable.

\section{Compliance with Ethical Standards}

Conflicts of Interest The authors declare that they have no conflicts of interest.

Ethical Approval Waived due to the retrospective and deidentified nature of this study.

Consent to Participate Waived due to the retrospective and deidentified nature of this study.

Consent for Publication Not applicable.

Code Availability Not applicable.

Open Access This article is licensed under a Creative Commons Attribution 4.0 International License, which permits use, sharing, adaptation, distribution and reproduction in any medium or format, as long as you give appropriate credit to the original author(s) and the source, provide a link to the Creative Commons licence, and indicate if changes were made. The images or other third party material in this article are included in the article's Creative Commons licence, unless indicated otherwise in a credit line to the material. If material is not included in the article's Creative Commons licence and your intended use is not permitted by statutory regulation or exceeds the permitted use, you will need to obtain permission directly from the copyright holder. To view a copy of this licence, visit http://creativecommons.org/licenses/by/4.0/.

\section{References}

1. Coutinho JM, Zuurbier SM, Gaartman AE, Dikstaal AA, Stam J, Middeldorp S, et al. Association between anemia and cerebral venous thrombosis. Stroke. 2015;46:2735-40. 
2. Luo Y, Tian X, Wang X. Diagnosis and treatment of cerebral venous thrombosis: a review. Front Aging Neurosci. 2018;10(2):115 .

3. Benifla M, Laughlin S, Tovar-Spinoza ZS, Rutka JT, Dirks PB. Unilateral postoperative deep cerebral venous thrombosis with complete recovery: a report of 2 cases. Pediatr Neurosurg. 2017:52:205-10.

4. Chung K, Tariq U, Khan RM, Nickles TP, Lock JH Jr. Unilateral thalamic venous infarction in an infant: a rare presentation of bilateral deep cerebral venous thrombosis. Case Rep Radiol. 2018: 3618619 .

5. Küker W, Schmidt F, Friese S, Block F, Weller M. Unilateral thalamic edema in internal cerebal vein thrombosis: is it mostly left? Cerebrovasc Dis. 2001;12:341-5.

6. Menon D, Sarojam MK, Gopal R. Unilateral thalamic infarct: a rare presentation of deep cerebral venous thrombosis. Ann Indian Acad Neurol. 2019;22:221-4.

7. Philpott C, Brotchie P. Unilateral internal cerebral vein thrombosis: review of literature and case illustration at 3 T. Eur J Radiol Extra. 2010;76:e1-6.

8. Beards SC, Yule S, Kassner A, Jackson A. Anatomical variation of cerebral venous drainage: the theoretical effect on jugular bulb blood samples. Anaesthesia. 1998;53:627-33.

9. Canhão P, Ferro JM, Lindgren AG, Bousser M, Stam J, Barinagarrementeria F. Causes and predictors of death in cerebral venous thrombosis. Stroke. 2005;36:1720-5.

10. Haug V, Linder-Lucht M, Zieger B, Korinthenberg R, Mall V, Mader I. Unilateral venous thalamic infarction in a child mimicking a thalamic tumor. J Child Neurol. 2009;24:105-9.

11. Mitaki S, Yamaguchi S. Efficacy of T2*-weighted gradient-echo MRI in early diagnosis of cerebral venous thrombosis with unilateral thalamic lesion. Case Rep Neurol Med. 2013:964650.

12. Sidek S, Rahmat K, Ramli N. Imaging findings of an isolated deep cerebral venous thrombosis in the absence of superficial sinus thrombosis. Neurology Asia. 2015;20(2):191-5.

13. Chung SW, Hwang SN, Min BK, Kwon JT, Nam TK, Lee BH. Unilateral thrombosis of a deep cerebral vein associated with transient unilateral thalamic edema. J Cerebrovasc Endovasc Neurosurg. 2012;14(3):233-6.

14. Forsting M, Krieger D, Seier U, Hacke W. Reversible bilateral thalamic lesions caused by primary internal cerebral vein thrombosis: a case report. J Neurol. 1989;236:484-6.
15. Herrmann KA, Sporer B, Yousry TA. Thrombosis of the internal cerebral vein associated with transient unilateral thalamic edema: a case report and review of the literature. AJNR Am J Neuroradiol. 2004;25:1351-5.

16. Leach JL, Fortuna RB, Jones BV, Gaskill-Shipley MF. Imaging of cerebral venous thrombosis: current techniques, spectrum of findings, and diagnostic pitfalls. RadioGraphics. 2006;26:S19-43.

17. Herrero M, Barcia C, Navarro JM. Functional anatomy of the thalamus and basal ganglia. Childs Nerv Syst. 2002;18:386-404.

18. Johnson MD, Ojemann GA. The role of the human thalamus in language and memory: evidence from electrophysiological studies. Brain Cogn. 2000;42:218-30.

19. Li S, Kumar Y, Gupta N, Abdelbaki A, Sahwney H, Kumar A, et al. Clinical and neuroimaging findings in thalamic territory infarctions: a review. J Neuroimaging. 2018;28:343-9.

20. Byrnes JR, Wolberg AS. Red blood cells in thrombosis. Blood. 2017;130(16):1795-9.

21. Escobedo J, Paz-Aragón E, Vega-Rodríguez LH, Sanfeliz MAB, Estrada-Rodríguez H, González-Geueroa E, et al. The methylenetetrahydrofolate reductase C677T (rs1801133) and apolipoprotein A5-1131T >c (rs662799) polymorphisms, and anemia are independent risk factors for ischemic stroke. J Stroke Cerebrovasc Dis. 2018;27(5):1357-62.

22. Walecki J, Mruk B, Nawrocka-Laskus E, Piliszek A, Przelaskowski A, Sklinda K. Neuroimaging of cerebral venous thrombosis (CVT) - old dilemma and the new diagnostic methods. Pol J Radiol. 2015;80:368-73.

23. Chiewvit P, Piyapittayanan S, Poungvarin N. Cerebral venous thrombosis: diagnosis dilemma. Neurol Int. 2001;3:e13.

24. Wetzel SG, Kirsch E, Stock KW, Kolbe M, Kaim A, Radue EW. Cerebral veins: comparative study of CT venography with intraarterial digital subtraction angiography. AJNR Am J Neuroradiol. 1999;20:249-55.

25. Tuttle C, Boto J, Martin S, Barnaure I, Korchi AM, Scheffler M, et al. Neuroimaging of acute and chronic unilateral and bilateral thalamic lesions. Insights into Imaging. 2019;10:24.

26. Saposnik G, Barinagarrementeria F, Brown RD Jr, Bushnell CD, Cucchiara B, Cushman M, et al. Diagnosis and management of cerebral venous thrombosis. Stroke. 2011;42:1158-92.

Publisher's Note Springer Nature remains neutral with regard to jurisdictional claims in published maps and institutional affiliations. 